\title{
UNA COMPARACIÓN A PARTIR DEL STIFFNESS TEST Y EL CMJ ENTRE DEPORTISTAS DE CHEERLEADING PROFESIONAL(SELECCIÓN COLOMBIA) Y AMATEUR.
}

\author{
A comparison from the Stiffness Test and the CMJ between professional Cheerleading (Colombia \\ Selection) and Amateur athletes.
}

Nicolle López

nlopez59@estudiantes.areandina.edu.co

Ricardo Cortes

rcortes6@estudiantes.areandina.edu.co

Cristian Yánez

cyanez@areandina.edu.co

Ana Masso

anmasso@areandina.edu.co

Fundación Universitaria del Área Andina. Profesional en entrenamiento deportivo

Colombia

\section{Resumen}

Uno de los movimientos técnicos más repetidos dentro de las sesiones de entrenamiento de la gimnasia y las acrobacias, y en el que se pueden alcanzar valores elevados de impacto contra el suelo es la recepción tras los saltos. Un aspecto diferenciador de esta especialidad gimnástica como lo es el Cheerleading es la poca utilización de componentes amortiguadores en el entrenamiento, por lo que la absorción de las fuerzas de reacción del suelo en el aterrizaje depende únicamente del componente activo de los deportistas (trabajo excéntrico de la cadena extensora del miembro inferior). Una mala técnica de amortiguación provocará que el porrista incremente el riesgo de padecer una lesión por sobrecarga. Métodos: Se realizó el Stiffness Test (ST) y el salto contra movimiento (CMJ) en 6 deportistas élite (Selección Colombia) y en 6 deportistas amateur de Cheerleading entre los $22 \pm 5$ años, con peso corporal 59 $\pm 10 \mathrm{~kg}$ y talla 1,64 \pm 1 , un IMC de 24,15 $\pm 3,5$ aparentemente sanas, físicamente activos y sin lesiones previas a la prueba. para evaluar la rigidez en el impacto de las articulaciones y la fatiga muscular en las extremidades inferiores en el Cheerleading. Propósito: Comparar las diferencias en los resultados de las diferentes variables (Pico de fuerza, altura de salto, tiempo de vuelo, fatiga muscular en miembros inferiores, entre otras) con respecto a los resultados del ST y el CMJ. entre de los deportistas aficionados y los deportistas élite (Selección Colombia). Resultados: La desviación estándar obtenida de la población a la que se le aplicó el Stiffness Test y el CMJ (6 atletas de la Selección Colombia de Cheerleading, 6 deportistas amateurs de Cheerleading) fue $22 \pm 5$ años, con peso

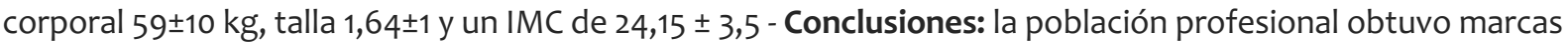
superiores en todas los aspectos según la hipótesis planteada, excepto en la variable (CRFD) que se expone como producto de la rigidez (LS) de la selección profesional. Es decir, que la rigidez permite, en este caso, orientar la mayor cantidad de energía en la fase excéntrica logrado así un efecto rebote donde no sea necesario agotar energía y reclutamiento en la fase concéntrica. Desde una perspectiva neuromuscular, la adaptabilidad del entrenamiento por alto rendimiento y experiencia hace que no se convierta necesario un alto nivel de desarrollo concéntrico de la fuerza, por ende se vuelve en un movimiento neuromuscularmente económico alcanzando igualmente resultados satisfactorios en la altura del salto.

Palabras clave: Cheerleading, élite, amateur, Stiffness Test, CMJ, lesión. 


\section{INTRODUCCIÓN}

El Cheerleading se ha convertido en una tendencia deportiva muy aclamada por los jóvenes (Hardy, I.; \& et al. 2017). El porrismo siendo un deporte excluyente en estados unidos y consolidado por diferentes estudios como un deporte de alto riesgo en universidades, colegios y escuelas presente a al impacto que tiene el cuerpo frente a la gimnasia, pirámides, baile y demás situaciones que avalen este deporte (Shields \& Smith, 2009), hoy en día es muy popular en diferentes lugares de Colombia. En este deporte y según las estadísticas presentes en estudios realizados en Estados Unidos, muestran un número de lesiones de cantidad superior, que, aunque en otros deportes es mayor, éste tiene una alta incidencia en lesiones (Shields \& Smith, 2009). Esto se debe a no entrenar de la manera adecuada, no realizar estudios para cada deportista, puede incidir en una lesión para éste por la falta de un buen manejo de cargas, desconocimiento o la falta de elementos de seguridad que generen la total seguridad que necesita las habilidades que se realizan en este deporte.

Por consiguiente, cheerleading es un deporte en el cual involucra bailes, pirámides, piruetas y lanzamientos siguiendo un ritmo con música formando una rutina (International cheer Union, 2019) El cheerleading requiere de bastante precisión. Los movimientos que van acompañados de cantos y exclamaciones consisten casi en su totalidad de la secuencia de movimientos. (López M. 2009) Siendo el movimiento parte de la técnica para una correcta ejecución.

\section{Método}

a) Población y muestra: Se aplicó el Stiffness Test y el CMJ a 6 atletas de la Selección Colombia de Cheerleading, 6 deportistas amateurs de Cheerleading entre 22 \pm 5 años, con peso corporal 59 $\pm 10 \mathrm{~kg}$ y talla 1,64 \pm 1 , un IMC de 24,15 $\pm 3,5$ aparentemente sanas, físicamente activos y sin lesiones previas a la prueba.

b) Diseño y protocolo: El método usado fue el Stiffness Test y el CMJ. La prueba se realizó en una plataforma de fuerza. Cada individuo fue registrado y se tomó el historial breve deportivo y datos barométricos previos a la prueba, como la talla, el peso, porcentaje graso, porcentaje muscular, edad metabólica y cantidad de grasa visceral.

Se informó a los participantes sobre el procedimiento a seguir y se hizo la entrega del consentimiento informado el cual leyeron y firmaron haciendo seguimiento de una investigación ética.

Para la realización del protocolo se utilizó una plataforma de fuerza.

Se inició con el Stiffness test en donde los sujetos a evaluar se familiarizaron con la prueba en sus tres condiciones (saltos con pierna dominante, contraria y juntas) en el cual se determinaron y estandarizaron 15 saltos con cada pierna y 15 saltos con las dos piernas al tiempo manteniéndose cerradas. A continuación, se realizó el CMJ en el cual se ejecutó 3 veces para determinar la fiabilidad de la prueba y así mismo promediar sus resultados de cada salto.

Respectivamente. Así, cada sujeto realizó seis pruebas de salto, con una duración de aproximadamente 20 seg por prueba. Inicialmente, los sujetos se encontraban erectos durante aproximadamente tres segundos y luego comenzaron saltando en su lugar hasta que se indique la finalización de cada procedimiento.

Para evitar la potencial influencia del uso de los brazos como modelo propulsión y margen de error, los sujetos realizaron la tarea saltando con sus manos colocadas lateralmente en su cintura.

Posteriormente, a los datos tomados se les inició su correspondiente análisis estadístico.

\section{Resultados}

La desviación estándar obtenida de la población a la que se le aplicó el Stiffness Test y el CMJ (6 atletas de la Selección Colombia de Cheerleading, 6 deportistas amateurs de Cheerleading) fue $22 \pm 5$ años, con peso corporal $59 \pm 10 \mathrm{~kg}$, talla $1,64 \pm 1$ y un IMC de $24,15 \pm 3,5$. 
Los resultados arrojaron que en la prueba de CMJ, el promedio de la altura del salto fue de 45,4 cm, promedio del time flight fue de 30,5, promedio pico de velocidad 2283,06 m/s, promedio de pico de poder fue 60,07 $\mathrm{W} / \mathrm{Kg}$. Por otra parte, las variables que se registraron en las fases concéntricas y excéntricas de los saltos arrojaron en promedio: Pico de fuerza concéntrico $1446 \mathrm{~N}$, pico de fuerza concéntrica 3649,87 N/Kg, Tasa concéntrica del desarrollo de la fuerza $1489,41 \mathrm{~N} / \mathrm{s}$, Tasa concéntrica del desarrollo de la fuerza 2,33 N/Kg/s, Tasa excéntrica del desarrollo de la fuerza 1748,84 N/s y Tasa excéntrica del desarrollo de la fuerza 2,98 $\mathrm{N} / \mathrm{Kg} / \mathrm{s}$.

Los resultados del CMJ aplicado en la población aficionada y profesional se evidencian en la siguiente tabla:

\begin{tabular}{|c|c|c|c|c|c|c|}
\hline Población & \begin{tabular}{|l} 
ALTURA \\
DE SALTO \\
$(\mathrm{cm})$
\end{tabular} & $\begin{array}{l}\text { PEAK } \\
\text { VELOCITY } \\
\text { (M/S) }\end{array}$ & \begin{tabular}{|l} 
PEAK \\
POWER \\
(W/Kg)
\end{tabular} & $\begin{array}{l}\text { PEAK } \\
\text { CONCENTR } \\
\text { IC FORCE } \\
(\mathrm{N} / \mathrm{Kg})\end{array}$ & $\begin{array}{l}\text { CONCENTRI } \\
\text { C RATE OF } \\
\text { FORCE } \\
\text { DEVELOP } \\
(\mathrm{N} / \mathrm{Kg} / \mathrm{s})\end{array}$ & \begin{tabular}{|l} 
ECCENTRIC \\
RATE OF \\
FORCE \\
DEVELOP \\
(N/Kg/s) \\
\end{tabular} \\
\hline Profesional & 36,23 & 2,61 & 58,37 & 2,28 & 1,29 & 1,95 \\
\hline Profesional & 30,28 & 2,30 & 53,51 & 2,37 & 1,05 & 3,21 \\
\hline Profesional & 34,25 & 2,73 & 72,11 & 2,69 & 2,84 & 3,51 \\
\hline Profesional & 36,42 & 2,57 & 69,62 & 2,76 & 1,46 & 3,29 \\
\hline Profesional & 23,94 & 2,19 & 50,42 & 2,36 & 0,68 & 2,59 \\
\hline Profesional & 22,51 & 2,15 & 50,03 & 2,37 & 0,68 & 2,43 \\
\hline amateur & 30,87 & 2,34 & 51,21 & 2,24 & 1,94 & 2,46 \\
\hline amateur & 28,07 & 2,39 & 49,38 & 2,10 & 0,73 & 1,58 \\
\hline amateur & 25,39 & 1,94 & 41,13 & 2,16 & 2,31 & 1,59 \\
\hline amateur & 35,43 & 2,69 & 58,76 & 2,23 & 1,79 & 2,37 \\
\hline amateur & 26,51 & 2,36 & 53,67 & 2,32 & 3,15 & 2,96 \\
\hline amateur & 33,21 & 2,62 & 54,17 & 2,11 & 0,60 & 1,54 \\
\hline
\end{tabular}

Tabla 1: Los resultados del CMJ aplicado en la población aficionada y profesional

Asimismo, sobre cada variable que se registra en el CMJ, a través de un diagrama de cajas y bigotes se identificó la ubicación de la población y su estado frente a cada variable:

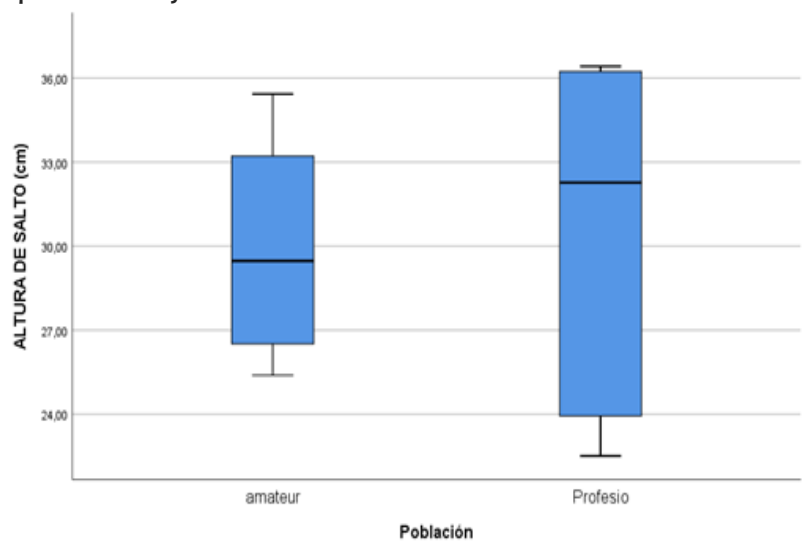

Figura 1: CMJ aplicado en la población aficionada y profesional

La variable de altura de salto $(\mathrm{cm})$ arrojó un promedio de 30,61cm (profesional), 29,91 (amateur), su respectiva mediana es 32,27 (profesional) y 29,47 (amateur).

La altura del salto mostró una diferencia no significativa ya que el promedio profesional fue $30,61 \mathrm{~cm}$ y el amateur de $29,91 \mathrm{~cm}$.

El pico de velocidad tampoco tiene una diferencia drástica pues el promedio profesional fue $2,43 \mathrm{~m} / \mathrm{s}$ y el amateur fue $2,39 \mathrm{~m} / \mathrm{s}$. Por otro lado, el pico de poder si arroja datos más relevantes, en este se evidencia una diferencia que puede indicar la potencia característica de la selección profesional: promedio profesional 59,01 $\mathrm{W} / \mathrm{Kg}$ y promedio amaterur 51,39 W/Kg.

Se sometieron los datos arrojados a estadística y normalidad utilizando la prueba no paramétrica Mann Whitney, en donde el resultado más significativo en cuanto a promedios fue hallado en la variable Peak concentric force con un valor de $\mathrm{P}=0,008$. 


\section{PEAK CONCENTRIC FORCE (N/Kg)}

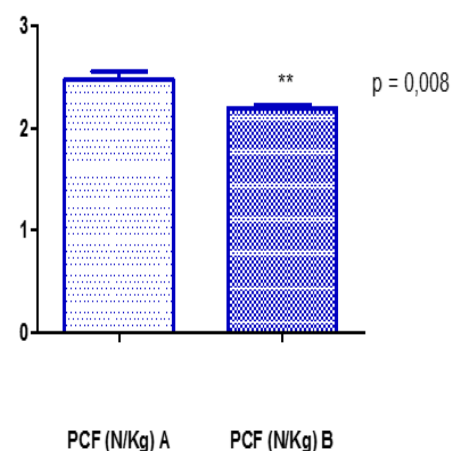

Figura 2: Pico de fuerza concéntrica de la población aficionada y profesional

El grupo A está referido a los deportistas profesionales y el B a los amateurs. El pico de fuerza concéntrica conlleva de igual manera con el resultado del pico de poder, este arrojó que el promedio para la población profesional fue de $2,47 \mathrm{~N} / \mathrm{Kg}$ por otro lado el promedio de la población amateur fue 2,19 N/Kg, siendo significativamente mayor.

\section{STIFNESS TEST}

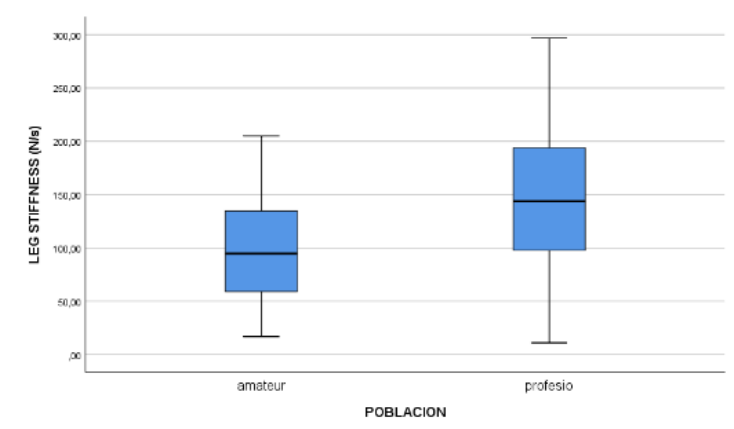

Figura 3: Rigidez de pierna población aficionada y profesional

Se hallaron diferencias estadísticamente significativas en la rigidez de la pierna del propio deportista variando entre pierna izquierda y pierna derecha, el tiempo de contacto variante entre la pierna dominante y no dominante (Izquierda y derecha)

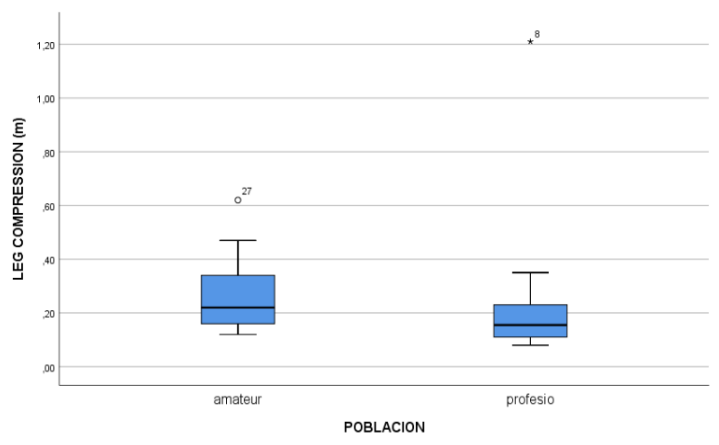

Figura 4: Compresión de pierna de la población aficionada y profesional 


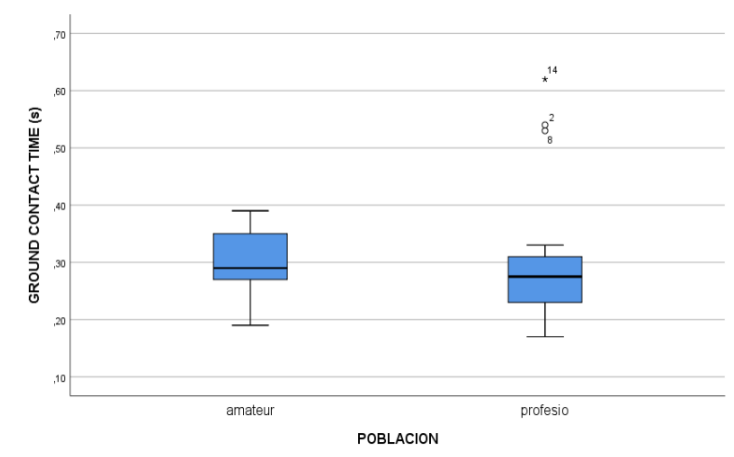

Figura 5: Tiempo de contacto del piso de la población aficionada y profesional

\section{Conclusión}

Las tasas concéntricas y excéntricas del desarrollo de la fuerza presentan variables previstas frente al resultado aparentemente esperado. El promedio profesional de la CRFD obtuvo un 1,86 (N/Kg/s) y el promedio amateur de la CRFD fue de $1,71(\mathrm{~N} / \mathrm{Kg} / \mathrm{s})$. Esto indica que la población aficionada posee niveles del desarrollo de la fuerza concéntrica menores que los de la población profesional. Finalmente, el resultado arrojado en el ERFD fue orientado y pronosticado según lo esperado, el promedio profesional fue de 2,83 $(\mathrm{N} / \mathrm{Kg} / \mathrm{s})$ y el promedio amateur fue de $2,08(\mathrm{~N} / \mathrm{Kg} / \mathrm{s})$, situándose así por encima de la media obtenida. En conclusión, la población profesional obtuvo marcas superiores en todos los aspectos según la hipótesis planteada, en la variable (PCF). Es decir, que la rigidez permita orientar la mayor cantidad de energía en la fase concéntrica logrado así un efecto rebote donde no sea necesario agotar energía y reclutamiento en la fase excéntrica. Desde una perspectiva neuromuscular, no se convierte necesario un alto nivel de desarrollo excéntrico de la fuerza, por ende se vuelve en un movimiento neuromuscularmente económico alcanzando igualmente resultados satisfactorios en la altura del salto.

Según los datos obtenidos se concluye que, por la ejecución del movimiento y la práctica deportiva repetitiva a nivel profesional, genera una estimulación, adaptación y optimización de la fase concéntrica de esta manera se produce mayores resultados en los picos de fuerza concéntrica.

En la técnica del deporte se encuentra movimientos donde se evidencia la ejecución de cortos movimientos excéntricos, favoreciendo la fuerza concéntrica generada por el gesto.

Puede justificarse que la habilidad del gesto deportivo genera adaptaciones de orden económico en cuanto al desempeño del salto, donde con el fin de que el éste sea técnico, simétrico, con menor gasto energético y con un desempeño óptimo (Economía del gesto).

Desarrollan muy buena potencia con una buena técnica.

Entrenar la saltabilidad y fuerza explosiva en porristas afirma que la rigidez puede influir positiva o negativamente, en este caso se recomendaría trabajar la flexibilidad con el fin de mejorar las variables de desempleo, aunque se destaca que con el Leg Stiffness disminuido logran resultados satisfactorios.

\section{Recomendaciones}

Se recomienda trabajar la flexibilidad y el componente pliométrico con el fin de mejorar el desempeño de las variables a través de los componentes elásticos paralelos y en serie del músculo (músculo, tendones y ligamentos).

\section{Referencias bibliográficas}

Angela Bagnulo, 2012. Cheerleading Injuries. The Journal of the Canadian Chiropractic association. PMC3501916 
Brauner, T., et al. (2013). Leg stiffness: Comparison between unilateral and bilateral hopping tasks. Human Movement Science.

BTS Bioengineering Corp. (2017). Protocol Leg Stiffness. Brooklyn, NY, USA

Claire, T., Farley, H., Ciska, VS., \& Louie, M. (1998). Mechanism of leg stiffness adjustment for hopping on surfaces of different stiffnesses Locomotion Laboratory, Department of Integrative Biology, University of California, Berkeley, California 94720-3140; and Department of Human Movement Sciences, Vrije Universiteit, 1081 BT Amsterdam, The Netherlands

Cormie P, McGuigan MR, Newton RU, (2010). Changes in the eccentric phase contribute to improved stretch-shorten cycle performance after training.

Croisier, J. (2004). Muscular imbalance and acute lower extremity muscle injuries in sport. International SportMed Journal, Vol. $5, \mathrm{n}^{\circ} 3$, 169-176.

Currie D \& et al. 2015. Lesiones de porristas en las escuelas secundarias de Estados Unidos. Pediatría. 2016 Ene; 137 (1). doi: 10.1542 / peds.2015-2447.

Frederick O. Mueller, 2009. Cheerleading Injuries and Safety. Journal of Athletic Training. PMC2775356

López M 2009. Cheerleading Technique Training Show. Sports Publishers Association.

McNitt-Gray, JL, T. Yokoi, y C. Millward. Aterrizaje ajustes de estrategia hechas por gimnastas en respuesta a la altura de caída y la composición estera. J. Appl. Biomech. 9: 173-190,

McNitt-Gray, JL, T. Yokoi, y C. Millward. Aterrizaje estrategias utilizadas por gimnastas en diferentes superficies. J. Appl. Biomech. 10: 237-252, 1994.

P. Grimshaw; \& et al. 2007. Sport and exercise biomechanics. Pág. 63

Riveros-Charry, R. (2012). Evaluación de tono muscular, Evaluación a pacientes. Buenos Aires: Guillete Children.

Rob Gathercole, Ben Sporer, Trent Stellingwerff, and Gord Sleivert, (2015). Alternative Countermovement-Jump Analysis to Quantify Acute Neuromuscular Fatigue.

Rodríguez, l., et al. (2010). "Evolución y relación de la capacidad de salto y amortiguación en gimnastas de rítmica de alto nivel". Cuadernos de psicología del deporte.

Roger M. Enoka 2008 Neuromechanics of human movement. Acquisitions editor.

Sastre, G. A. (2012). Le facteur influençant la flexibilité musculaire et sa relation avec des altérations posturales des altérations posturales. General José María Córdova, Vol 10 , Núm 10, Año.

Vaz, D. V. (2006). Análise da relação entre flexibilidade e rigidez passiva dos isquiotibiais. Fisioterapia e pesquisa, 13(2), 71-82.

Villarreal, e. s.-s., Kellis, e., Kraemer, w. j., \& Izquierdo, m. (2009).Determining variables of plyometric training for improving vertical jump height performance: ameta-analysis. Studies and sport medicine center, vol 22 num 3.

Walker B. 2010. La anatomía de las lesiones deportivas. Editorial Paidotribo.

Yánez, C. A. (2016). Variables bioquímicas y del desempeño del salto contra movimiento para monitorizar la fatiga neuromuscular en deportistas de resistencia. Bogotá: Repositorio. Universidad Nacional de Colombia. 